\title{
ESTUDIO COMPARATIVO DE LOS PROCESOS DE EVALUACIÓN A NIVEL SUPERIOR ENTRE PANAMÁ Y COSTA RICA.
}

\author{
COMPARATIVE STUDY OF THE EVALUATION PROCESSES AT THE HIGHER \\ LEVEL BETWEEN PANAMA AND COSTA RICA.
}

José F. Prado ${ }^{1}$. josefelixprado27@ gmail.com. https://orcid.org/0000-0002-3101-606X.

Ana Álvarez de Barrios 2 . ana-m.alvarez@up.ac.pa. https://orcid.org/0000-0002-0606-1424. Virgilio M. Sousa ${ }^{3}$. virgilio.sousa-v@up.ac.pa. https://orcid.org/0000-0001-7812-110X

\section{Universidad de Panamá. Panamá, Panamá}

Resumen: Las universidades constituyen una herramienta clave para el desarrollo de un país; velar por la calidad que ofrecen es un elemento importante de su proyección internacional. En ese sentido, la evaluación y la autoevaluación institucional responden a las demandas para lograr que las universidades estén acordes con los avances de la sociedad y sean también un reflejo de ella. Este estudio responde a un enfoque documental, ya que se basa en otras investigaciones y su veracidad procede de informes o investigaciones realizadas previamente, y su prueba se justifica en documentos oficiales auténticos, referidos a la población en estudio. Es cualitativo bajo un diseño descriptivo que detalla características, fenómenos y situaciones de una población específica (Pérez 2001). Dio lugar a comparar constructos que permitieron comprender la importancia de las investigaciones realizadas por los distintos países mediante la observación y estudio de casos. La población o universo al cual esta dirigida esta investigación, la constituyen los Sistemas de Evaluación a nivel Superior entre Panamá y Costa Rica. La muestra del estudio ha considerado la situación de los procesos de Evaluación de Costa Rica y Panamá. El Estudio comparativo de los procesos de evaluación de Costa Rica y Panamá constituyo un análisis amplio de los procesos que iniciaron respectivamente en 1999 y 2011. Por ende, Costa Rica lleva doce años por delante de Panamá en experiencia realizando estos procesos, por lo que pudimos observar de las normativas, informes y reglamentos aparenta ser más ordenado que nuestro país. Cada país ha llevado adelante sus procesos y las cifras demuestran que los resultados que han tenido son positivos. Los procesos de evaluación y acreditación de la educación superior son instrumentos para promover el mejoramiento de la calidad de las instituciones de educación superior y de sus programas, así como para ofrecer información pertinente a la sociedad, sobre la calidad de los mismos.

Palabras claves: Evaluación, acreditación, calidad, educación superior, mejoramiento.

\footnotetext{
Summary

Universities are a key tool for the development of a country; ensuring the quality they offer is an important element of their international projection. In this sense, institutional evaluation and self-evaluation respond to the demands to ensure that universities are in line with the advances of society and are also a reflection of it. This study responds to a documentary approach, since it is based on other investigations and its veracity comes from reports or investigations carried out previously, and its proof is justified in authentic official documents, referring to the population under study. It is qualitative under a descriptive design that details characteristics, phenomena and situations of a specific population (Pérez 2001). It led to the comparison of constructs that allowed us to understand the importance
} 
of the research carried out by the different countries through observation and case studies. The population or universe to which this research is directed is constituted by the Higher Level Evaluation Systems between Panama and Costa Rica. The study sample has considered the situation of the Evaluation processes of Costa Rica and Panama. The Comparative Study of the evaluation processes of Costa Rica and Panama constituted a broad analysis of the processes that began respectively in 1999 and 2011.

Therefore, Costa Rica has been twelve years ahead of Panama in experience carrying out these processes, so we could observe the rules, reports and regulations that appear to be more orderly than our country. Each country has carried out its processes and the figures show that the results they have had are positive. The higher education evaluation and accreditation processes are instruments to promote the improvement of the quality of higher education institutions and their programs, as well as to offer pertinent information to society on their quality.

Key words: Evaluation, accreditation, quality, higher education, improvement.

\section{Introducción.}

En las últimas décadas, en América Latina y Centroamérica y en particular en Panamá y Costa Rica, se han dado cambios significativos en los sistemas de educación. Hablar el mejoramiento de la calidad de la educación, ha llevado a los gobiernos a establecer un marco legal apropiado para la implementación de sistemas de evaluación y acreditación de los servicios de educación. El primer Sistema de Supervisión y Mejoramiento de la Calidad de las Instituciones de Educación Superior en América Central, se creó en El Salvador, en 1997 y, luego evolucionó a lo que es hoy el Consejo de Acreditación CdA de El Salvador. Costa Rica creó el Sistema Nacional de Acreditación de la Educación Superior SINAES, el cual ya ha sido acreditado por una agencia de segundo nivel: el Consejo Centroamericano de Acreditación de la Educación Superior CCA. (Tünnermann 2008)

Este análisis comparativo permite ampliar la visión del camino recorrido en el tema de procesos de Evaluación a nivel Superior entre Panamá y Costa Rica. Como profesionales en el área de la educación reconocemos la evaluación como herramienta fundamental para la mejora continua y constante, mediante la autoevaluación, evaluación externa y seguimiento para el logro de la calidad de la educación.

\section{PARTE EXPERIMENTAL}

El estudio es documental ya que se basa en otras investigaciones y su veracidad procede de informes o investigaciones realizadas previamente, y su prueba se justifica en documentos oficiales auténticos, referidos a la población en estudio. Es cualitativo bajo un diseño descriptivo que detalla características, fenómenos y situaciones de una población específica (Pérez 2001). Dio lugar a comparar constructos que permitieron comprender la importancia de las investigaciones realizadas por los distintos países mediante la observación y estudio de casos.

La poblacion o universo al cual esta dirigida esta investigacion, la constituyen los Sistemas de Evaluación a nivel Superior entre Panamá y Costa Rica. La muestra del estudio ha considerado la situación de los proceso de Evaluación de Costa Rica y Panamá.

Las técnicas e instrumentos de recolección y análisis de datos se realizaron mediante:

- Estrategia de indagación

Se identificaron, caracterizaron y compararon las fuentes y enfoques teóricos que sustentan y caracterizaron la recopilación y análisis de los modelos de evaluación de los procesos de aprendizaje. 
Para ello, se realizaron las actividades de indagación las cuales requieren, entre otros aspectos; destrezas para identificar conceptos, suposiciones, teorías, el uso del pensamiento lógico, crítico, reflexivo, y las explicaciones alternativas. (Camacho, Castillo, \& de Franco, 2018)

- Análisis de Contenido

Se seleccionó una estrategia de lectura interpretativa, ya que, por una parte, se buscó captar el contenido íntegro de los textos al tratarse de informes de país, pero, por otra, también se buscó intentar obtener contenidos ocultos mediante la deducción y análisis del contenido manifiesto de los textos.

El procedimiento empleado para la recopilación y análisis comparativo de los procesos de Evaluación a nivel Superior entre Panamá y Costa Rica se desarrolló en cuatro etapas, las cuales son:

1. Planificación de la investigación: en esta etapa se realizó la búsqueda de antecedentes que permitieran datos bibliográficos que aportaran datos acerca de la importancia y relevancia del estudio. También se abordaron los aspectos de la sustentación teórica y el marco metodológico.

2. Colección, sistematización y análisis de los datos

3. Generación del informe de investigación

4. Presentación del resultado de la investigación

\section{Resultados y Discusión}

1. Situación Actual

La investigación realiza un estudio comparativo de los procesos de Evaluación a nivel Superior entre Panamá y Costa Rica. La evaluación en educación superior se basa principalmente en la implementación de estrategias, para mejorar la planeación educativa propuesta, no dejando de lado la importancia de logro de los objetivos propuestos para alcanzar el currículo y al mismo tiempo desarrollar las competencias de formas válidas y fiables, pues esto implica grandes cambios de enseñanza por la demanda de las nuevas necesidades sociales. (Rosales, 2019) Panamá y Costa Rica poseen un sistema de educación superior compuesto de universidades particulares y universidades oficiales, ambos países cuentan con un Sistema Nacional de Acreditación, en Costa Rica se denomina Sistema Nacional de Acreditación de la Educación Superior (SINAES) y en Panamá, el Sistema Nacional de Evaluación y Acreditación para el Mejoramiento de la Calidad de la Educación Superior Universitaria de Panamá. En Costa Rica el Sistema está compuesto por instituciones miembros las cuales se subdividen en, el Consejo y Profesionales Externos y en Panamá lo componen el Ministerio de Educación, el Consejo Nacional de Evaluación y Acreditación de la Educación Superior Universitaria de Panamá, la Comisión Técnica de Desarrollo Académico, las instituciones de educación superior universitarias oficiales y particulares que operan legalmente en la República de Panamá, el Consejo de Rectores de Panamá, como órgano de consulta y la Asociación de Universidades Privadas de Panamá, como órgano de consulta.

En ambos países la acreditación oficial solo la otorga la Agencia Acreditadora del Estado. Una de las diferencias es que en Costa Rica el SINAES comenzó a funcionar en el año de 1999 con el proceso de acreditación con carreras y programas, y en Panamá en el año 2011 a nivel institucional y posteriormente con las carreras y programas. El Consejo del SINAES, es el órgano de máxima decisión, está integrado por ocho ciudadanos que deben cumplir con los siguientes requisitos: tener la categoría de catedrático universitario o su equivalente, grado académico de maestría o doctorado, un mínimo de ocho años de experiencia docente en alguna universidad autorizada en el país. De los ocho miembros, cuatro serán elegidos por las universidades estatales y las universidades particulares designarán a los cuatro representantes restantes. Cobran una dieta por cada sesión a la que asistan por un máximo de 8 al mes. (SINAES, 2019)

Por el contrario, en Panamá el artículo 21 de la Ley 52 de junio de 2015, indica que el Consejo Nacional de Evaluación y Acreditación Universitaria de Panamá estará integrado por once 
miembros ad honorem, que representan a los diferentes sectores vinculados con el desarrollo y la transformación de la educación superior universitaria del país, así: 1. El ministro de Educación o su representante, quien lo presidirá, con derecho a voz y voto solo para desempatar, 2. El ministro de Economía y finanzas o su representante, 3. El secretario nacional de Ciencia, Tecnología e Innovación o su representante, 4. El presidente de la Comisión de Educación, Cultura y Deportes de la Asamblea Nacional o su representante, 5. El presidente de la Comisión Técnica de Desarrollo Académico o su representante, 6. El secretario ejecutivo del Consejo Nacional de Evaluación y Acreditación Universitaria de Panamá, solo con derecho a voz, 7. Dos miembros de las universidades oficiales o sus representantes, 8. Dos miembros de las universidades particulares o su representante, 9. Un miembro del Consejo Nacional de la Empresa privada por un periodo no mayor de dos años elegido entre ellos y de manera rotativa, 10. Un miembro de las organizaciones de profesionales de Panamá elegido entre ellos por un periodo no mayor de dos años y de manera rotativa, 11.Un miembro del Consejo Nacional de Educación.

Estos deberán cumplir con un perfil académico y conocimiento de la realidad del país, los cuales no están estipulados, los requisitos están normados para el cargo de secretario Ejecutivo del Consejo y no para los miembros de este.

En Costa Rica los costos del proceso de acreditación, la fase de evaluación externa tiene un costo de 7.300 dólares, se paga una sola vez cada 4 años. La revisión del informe de avance de cumplimiento tiene un costo de 500 dólares, por lo general se hace una vez al año. Esas tarifas han permanecido invariables desde que SINAES comenzó funciones en 1999. El Estado costarricense asume $2 / 3$ partes del costo total del proceso de acreditación, el tercio restante se cubre con el aporte de las instituciones de educación superior. (SINAES, 2019)

En Panamá, para el proceso de acreditación del año 2012 se cobraba un aproximado de B/.16426.18.00 pagadero en 3 tiempos: a la firma del acuerdo de compromiso, a la entrega del informe de autoevaluación y plan de mejoramiento al CONEAUPA y al momento de recibir el informe final de los pares por el CONEAUPA. (Acuerdo $N^{\circ} 03-2012$ )

\section{República de Panamá}

En Panamá, de acuerdo con el Censo Nacional de Población, en el año 2010 habían aproximadamente 1.81 millones de panameños con 25 años y más de edad (INEC, 2011). De esta cantidad:

- 1 millón (56\%) no tenían título que certifique algún tipo de estudio.

- $\quad 486,550(27 \%)$ solo han completado educación secundaria o tenían estudios

universitarios incompletos

- $\quad 217,039$ (12\%) tenían título de licenciatura en ese momento.

- $\quad 35,629(<2 \%)$ tenían título de técnicos universitarios

- $\quad 41,094(<3 \%)$ terminaron estudios de posgrado o maestría.

- $2736(0,15 \%)$ terminaron el doctorado

El Censo de Población debió realizarse en el mes de mayo de 2020, este proceso se vio afectado por la pandemia de la COVID-19, por lo que no se cuenta con cifras actualizadas de esta temática y sólo las universidades oficiales reportan su matrícula al Instituto de Estadística y Censo de la Contraloría General de la República de Panamá.

\subsection{Aspectos Generales de la Educación Superior en Panamá}

Con el establecimiento del Consejo de Rectores en el año de 1995 se dio el inició al proceso de establecimiento de un Consejo Nacional de Acreditación de la Educación Superior. Lo que finalmente se logra con la promulgación de la Ley 30 de 2006 se crea el Consejo Nacional de Evaluación y Acreditación Universitaria de Panamá, identificado con las siglas CONEAUPA, como un organismo evaluador y acreditador, rector del Sistema Nacional de Evaluación y Acreditación para el Mejoramiento de la Calidad de la Educación Superior Universitaria. El 
proceso fue un gran reto para la Secretaría Ejecutiva del CONEAUPA, porque tuvieron que cumplir con muchos aspectos: elaboración de la matriz y reglamentos de la acreditación, sensibilización nacional sobre el tema a las universidades, comunidad universitaria, así como al personal interno y los pares observadores por Panamá y por Latinoamérica. (Barrios, Castillero, \& Sousa, 2020)

\subsection{El Sistema Nacional de Evaluación y Acreditación para el Mejoramiento de la Calidad de la Educación Superior Universitaria de Panamá}

En Panamá, el Sistema Nacional de Evaluación y Acreditación para el Mejoramiento de la Calidad de la Educación Superior Universitaria de Panamá fue creado en el artículo 1 de la Ley 52 de junio de 2015 este sistema es creado como un organismo con autonomía académica, personería jurídica y patrimonio propio sujeto a la orientación y política general del Organo Ejecutivo, adscrito al Ministerio de Educación, no existe un espacio en la República de Panamá al que uno asista y sea la sede del Sistema Nacional de Evaluación y Acreditación para el Mejoramiento de la Calidad de la Educación Superior Universitaria de Panamá. (Ley $\mathrm{N}^{\circ}$ 52, 2015). Con la Ley 30 de 2006 y posteriormente la Ley 52 las únicas nuevas instituciones que surgieron fueron el Consejo Nacional de Evaluación y Acreditación de la Educación Superior Universitaria de Panamá y la Comisión Técnica de Desarrollo Académico.

\subsection{Proceso de Acreditación}

En Panamá, los procesos de Evaluación y Acreditación para el Mejoramiento de la Calidad de la Educación Superior Universitaria se iniciaron en el año 2011, con la Matriz de Evaluación como base para que las Universidades cumplieran con los factores e indicadores según su categoría, en el Siglo XXI. (Barrios, Castillero, \& Sousa, 2020). De la primera experiencia de acreditación se obtuvieron 5 universidades oficiales acreditadas y 4 carreras de ciencias de la salud de universidades oficiales acreditadas. Por el lado del sector particular, se acreditaron 18 universidades particulares y 5 carreras de ciencias de la salud de universidades particulares.

\subsection{Modelo de Evaluación}

El Modelo de Evaluación con fines de acreditación institucional fue aprobado en Sesión Extraordinaria VIII del 6 de diciembre de 2019 por el Pleno del CONEAUPA se basa en un enfoque sistémico, orientado a la evaluación con fines de acreditación institucional. Está basado en los principios que rigen el Sistema Nacional de evaluación y Acreditación para el Mejoramiento de la Calidad de la Educación los cuales se encuentran estipulados en el artículo 5 de la Ley 52 de 2015 y son el mejoramiento continuo de la calidad, respeto a la autonomía universitaria, reconocimiento de la diversidad de las instituciones universitarias y sus diferentes programas y modalidades de enseñanza; tratamiento justo y equitativo de las instituciones de educación superior universitaria, transparencia en el cumplimiento con todas las reglas éticas, morales y normas jurídicas que regulan la educación superior y confidencialidad en el manejo de la información. (Ley $\mathrm{N}^{\circ}$ 52, 2015)

La igualdad y la comparabilidad se constituyen como los principales pilares del Modelo de Evaluación del Sistema Nacional de Evaluación y Acreditación para el Mejoramiento de la Calidad de la Educación, igualdad en el tratamiento de las instituciones universitarias y comparabilidad comprendida como el resultado de enfrentar procesos iguales regidos por políticas idénticas y compararlos. El modelo contempla la evaluación mediante tres fases: autoevaluación institucional o de programas, evaluación externa y acreditación.

\subsubsection{Evolución del Modelo}

El modelo presentado en el año 2019 se ha mantenido al igual que en el modelo del 2012 con un enfoque sistémico, el cual propone soluciones en las cuales se tienen que considerar diversos elementos y relaciones que conforman las partes intervinientes de todo sistema y el entorno del cual forma parte. (Terlizzi, 2014). En cuanto los aspectos que contempla el modelo se pueden observar como ha variado del 2012 al 2019 en la tabla 1. 
De esto es importante destacar que para el año 2019 desaparece el aspecto obligatorio en el modelo de evaluación, a pesar de que el artículo 10 de la Ley 52 de junio de 2015 claramente indica que los procesos de acreditación institucional serán obligatorios para todas las universidades con más de ocho años de su creación, no obstante, pueden incorporarse de manera voluntaria antes de completar este periodo. (Ley $\mathrm{N}^{\circ}$ 52, 2015). Como parte de la investigación se ha realizado la compilación de la matriz institucional del año 2012 la cual utilizó como factores: la docencia universitaria, investigación e innovación, extensión universitaria y gestión institucional universitaria.

Se realizó un análisis de la evolución de la matriz institucional destacando principalmente el cambio de nombre de los 4 factores los cuales en la matriz actual los denomina como: proyecto institucional, proyecto académico, comunidad universitaria e infraestructura. Estos aspectos son importantes, toda vez que, el artículo 4 de la Ley 52 de 26 de junio de 2015, indica que la docencia universitaria es una de las áreas indispensable en el análisis de los procesos de evaluación y acreditación, además define la acreditación como el procedimiento mediante el cual el un organismo autorizado reconoce formalmente que una institución de educación superior universitaria es competente para realizar las funciones esenciales de docencia, investigación, extensión y gestión. (Ley $N^{\circ}$ 52, 2015). Por lo que nos extraña de sobremanera el cambio de los nombres de los factores en este Modelo 2019, ya que no van acorde a lo contemplando en la normativa actual, aunado a que no se puede dejar de mencionar la reducción considerable de indicadores y estándares los cuales consideramos necesarios para la adecuada calidad de la educación superior panameña, teniendo la nueva matriz un total de 109 indicadores y estándares y en el 2012 se evaluaron 185.

También existe una variación en cuanto a la escala de valoración para la evaluación institucional, ya que de acuerdo con el artículo 23 del Decreto Ejecutivo $\mathrm{N}^{\circ} 539$ de 2018, se permite valorar y validar cualitativamente y cuantitativamente las características de una institución, carreras y programas, atendiendo a su entorno, de acuerdo con estándares de calidad previamente establecidos por el CONEAUPA. (Decreto $N^{\circ}$ 539, 2018). Para ello, CONEAUPA realizó la siguiente escala valorativa cualitativa y cuantitativa de la siguiente manera: cumple totalmente -5 , Cumple satisfactoriamente - 4, Cumple parcialmente- 3, Cumple escasamente- 2, No cumple - 1. (CONEAUPA,2020)

\subsubsection{Instrumentos de Evaluación}

En cumplimiento con lo establecido en las normas legales vigentes, el Consejo Nacional de Evaluación y Acreditación Universitaria de Panamá, aprueba en Sesión Extraordinaria VIII de 6 de diciembre de 2019, los Fundamentos del Modelo de Evaluación con fines de Acreditación Institucional la cual incluye cuatro factores: proyecto institucional, proyecto académico, comunidad universitaria e infraestuctura.

\subsubsection{Evaluadores}

La 52 de 26 de junio de 2015, define como Par Académico Externo, (homólogo o semejante) al experto nacional o internacional con solvenciamorla, ética y con probada idoneidad, trayectoria en su área de especialidad, respetado y reconocido por la comunidad profesional, científica o académica, poseedor de experiencia en la docencia y la gestión académica e institucional de la educación superior. Responsable de la evaluación externa, con total independencia, transparencia, imparcialidad y objetividad en los procesos de evaluación institucional o programa. La evaluación externa es la segunda etapa en el proceso de Acreditación, una vez se realiza el Informe de Autoevaluación por parte de la Institución que se acoge al proceso. Es en esta etapa que se incorporan los pares académicos externos. Los pares hacen una verificación tomando en cuenta los documentos presentados por la entidad y presentan juicios de calidad basados en sus observaciones e incluso en aquellos aspectos que pudieran no ser considerados en la autoevaluación y que resulten importantes y relevantes para 
apreciar la calidad de las instituciones en un campo específico. (CONEAUPA, 2020)

\subsubsection{Fases del Proceso}

Las fases del proceso de acreditación en Panamá se encuentran claramente definidas en el artículo 4 de la Ley 52 de 26 de junio de 2015 de la siguiente manera:

Autoevaluación o Evaluación interna: Es el proceso mediante el cual la institución de educación superior universitaria y sus integrantes asumen la responsabilidad de evaluar y analizar los logros como un todo a nivel institucional, así como los aspectos críticos de un programa determinado, con el objetivo de elaborar planes de mejora según los criterios delCONEAUPA. (Ley $\mathrm{N}^{\circ}$ 52, 2015)

Evaluación Externa: Proceso en el que un grupo de especialistas evaluadores, llamados pares académicos externos, realizan una verificación del informe de autoevaluación institucional o de programas, del plan de mejoras y de las condiciones internas de operación, el cual concluye con el informe final. (Ley $\mathrm{N}^{\circ}$ 52, 2015)

Acreditación: Proceso mediante el cual se reconoce de manera formal que la institución educativa superior universitaria es competente para realizar las funciones esenciales de docencia, investigación, extensión y gestión. (Ley N No 52, 2015)

\subsubsection{Beneficios de la Acreditación}

El proceso de acreditación en Panamá realizó un filtro en el sistema de educación superior panameña, donde solo han podido seguir funcionando las que cumplen con los estándares de calidad que exige CONEAUPA, a la fecha se tienen trece universidades cerradas y 30 en funcionamiento, cifras muy distintas a las que se manejaban al inicio de los procesos en el 2011. El Factor Investigación, se constituyó como un elemento importante dentro de la acreditación, se formaron semilleros de investigación y se incentivó la elaboración de trabajos de investigación como opción de graduación, se crearon diversas publicaciones, valoración de los docentes con estudios a nivel de doctorado.

El personal de las universidades panameñas ha recibido producto de la acreditación oportunidades de desarrollo profesional ofrecido al personal administrativo. De manera que se valide que las contrataciones del personal administrativo este en correspondencia con su formación académica y experiencia profesional. (Barrios, Castillero, \& Sousa, 2020)

\subsubsection{Incentivos de la Acreditación}

Los incentivos para aquellas universidades que obtuvieron su certificado de acreditación en la nueva legislación son el otorgamiento de incentivos de parte del Consejo Nacional de Evaluación y Acreditación Universitaria de Panamá para la gestión y realización de las funciones universitarias de investigación, extensión, docencia, gestión, innovación y movilidad académica, exoneración de impuestos exonerará los impuestos fiscales y municipales, préstamos a bajas tasas de interés a través de los bancos estatales y servicio de franquicia postal. (Ley No 52, 2015)

\subsubsection{La Reacreditación}

La reacreditación no estaba contemplada en la Ley 30 de junio de 2006, ya que era el inicio de los procesos de acreditación, sin embargo al ver que las acreditaciones tenían una vigencia de 6 años era necesario contemplar la reacreditación para aquellas universidades a las cuales sus acreditaciones fueran perdiendo vigencia. El Decreto 539 le dedica el capítulo V en su totalidad compuesto por tres artículos, que definen el proceso de reacreditación como la renovación de la acreditación institucional, de carrera o programas emitida por el Consejo Nacional de Evaluación y Acreditación Universitaria de Panamá (CONEAUPA), se orienta sobre el principio de calidad y mejoramiento continuo, fundado en el plan de mejoramiento institucional de carreras y programas. (Decreto $\mathrm{N}^{\mathrm{o}} 539,2018$ ). La normativa indica que el Consejo Nacional de 
Evaluación y Acreditación Universitaria de Panamá (CONEAUPA), elaborará los procedimientos y manuales pertinentes para el desarrollo de los procesos de reacreditación, hasta el momento no encontramos información sobre que estas guías se encuentren aprobadas para iniciar estos procesos, por lo que las universidades seguirán con sus acreditaciones con vigencia de seis años por un periodo más largo.

\section{República de Costa Rica}

Según diversos informes, Costa Rica se encuentra en el cuarto lugar entre 94 países con más accesibilidad para estudiar. Estos estudios demuestran que la educación en el país abarca en su totalidad, ya que el índice de las personas que saben leer y escribir es de $90.27 \%$ y el analfabetismo es de un $9.73 \%$ que equivale a 334,000 personas que no saben leer, ni escribir. El Sistema de Educación Superior (SES) estatal de Costa Rica aspira a constituirse en "referente de toda América Latina" (Plan Nacional de la Educación Superior Universitaria Estatal, PLANES 2011-2015), en tanto que "espacio estratégico de articulación caracterizado por la excelencia, la pertinencia, la oportunidad...", empleando como instrumentos el fomento de "múltiples interrelaciones en las áreas de docencia, investigación, extensión y acción social".

\subsection{Aspectos generales de la Educación Superior Costarricense}

La Constitución Política de Costa Rica, señala que las instituciones de educación superior universitaria estatal gozan de independencia para el desempeño de sus funciones y de plena capacidad jurídica para adquirir derechos y contraer obligaciones, así como para darse organización y gobierno propios... El Estado las dotará de patrimonio propio y colaborará en su financiación. (Art. 84). El Estado dotará de patrimonio propio a la Universidad de Costa Rica, al Instituto Tecnológico de Costa Rica, a la Universidad Nacional y a la Universidad Estatal a Distancia y les creará rentas propias, independientemente de las originadas en estas instituciones. Además, mantendrá - con las rentas actuales y otras que sean necesarias- un fondo especial para el financiamiento de la Educación Superior Estatal. Actualmente, existen hay 64 universidades (5 públicas y 53 privadas) y 6 internacionales con una oferta que sobrepasa las 1,400 carreras. Esta realidad, ubica a Costa Rica es el país con mayor número de estos centros de estudio per cápita a nivel latinoamericano y de ellas a octubre del 2011, solo 64 cuentan con Acreditación Oficial vigente del SINAES y 25 han obtenido la Reacreditación. (SINAES, 2011). La Tipología de la educación costarricense se estructura de la siguiente forma:

- Instituciones universitarias estatales: la Universidad de Costa Rica (1940), el Instituto Tecnológico de Costa Rica (1971), la Universidad Nacional (1973), la Universidad Estatal a Distancia (1977) y la Universidad Técnica Nacional (2008).UCR, ITCR, UNA, UNED.

- Instituciones parauniversitarias estatales: ECAG, CUC, CUP, CUNA, CIPET, CURDTS, CUNLIMON.

- Instituciones universitarias privadas: ............ 53

- Instituciones parauniversitarias privadas: .......34

- Instituciones universitarias internacionales:..... 6

\subsection{El Sistema Nacional de Acreditación de la Educación Superior}

En Centroamérica, el Consejo Superior Universitario Centroamericano (CSUCA) ha sido el organismo pionero no solo de la integración regional de la educación superior sino también de los procesos de aseguramiento de la calidad académica. El Primer "Plan para la integración regional de la educación superior centroamericana", aprobado por las universidades miembros del CSUCA en 1962. Lo cual ha conducido a la creación del Sistema de Carreras y Postgrados Regionales (SICAR) y, a partir de 1998, del Sistema Centroamericano de Evaluación y Acreditación de la Educación Superior (SICEVAES).

a. Número de Carreras Universidades Oficiales y tipo de acreditación otorgada: Costa Rica cuenta con cinco (5) universidades oficiales, de las 166 carreras que cuentan con el 
sello de SINAES, 97 pertenecen a universidades públicas. De las cinco universidades públicas agrupadas en el CONARE.

b. Número de Carreras de Universidades Particulares y tipo de acreditación otorgada: Es importante destacar que once universidades privadas (de las 54 existentes, todas asociadas a la UNIRE), cuatro universidades internacionales (CATIE, EARTH, Universidad para la Paz y recientemente la Universidad de San Carlos de Guatemala) y tres instituciones de educación parauniversitaria (Invenio, Escuela Técnica Agrícola e Industrial y CUC).

De las 166 carreras que cuentan con el sello de SINAES, 97 pertenecen a universidades públicas, mientras que 51 a privadas. 12 corresponden a Universidades Internacionales con sede en Costa Rica, 3 a Universidades Internacionales con sede fuera de Costa Rica, 2 a Parauniversitaria privada y 1 a Parauniversitaria pública.

\subsection{Proceso de Acreditación en Costa Rica.}

La Acreditación es un proceso por el cual una institución educativa evalúa periódicamente sus actividades educacionales y busca un juicio independiente, a través de pares académicos, sobre el logro de Objetivos educativos y Criterios de calidad establecidos por la institución acreditadora. (Young, Chambers \& Kells, 1983).

El SINAES realiza procesos de acreditación para carreras parauniversitarias, de grado y programas de postgrado. Un requisito de admisibilidad para que una carrera parauniversitaria, de grado o de postgrado se someta al proceso de evaluación con fines de acreditación es que se imparta en una institución educativa que haya formalizado su compromiso con los principios de calidad del SINAES. La acreditación oficial constituye un proceso permanente de fortalecimiento de la calidad de la educación superior que realizan en forma conjunta la carrera, la institución educativa y el SINAES, bajo el marco de un modelo de evaluación y que comprende las fases de: autoevaluación, evaluación externa y mejoramiento continuo.

La autoevaluación es un proceso de reflexión interna que realizan los actores involucrados en la carrera: académicos, investigadores, administradores, estudiantes, egresados, colegios profesionales universitarios y empleadores, entre otros. La evaluación externa está a cargo de tres expertos en el área objeto de evaluación, dos de esos expertos son internacionales y uno es nacional. La evaluación externa busca, por una parte, la validación que realizan pares académicos externos tanto de la etapa de autoevaluación como del informe resultante de ésta y por otra parte, la evaluación directa e "in situ" de la carrera o programa. La etapa de mejoramiento continuo comprende la valoración final de la calidad de la carrera que realiza el Consejo Nacional de Acreditación del SINAES mediante un proceso de triangulación que considera el Informe Final de Evaluación Externa, el Informe de Autoevaluación, el Compromiso de Mejoramiento y toda otra información obtenida a lo largo de las diversas etapas, fases y actividades del proceso. Finalmente, comprende también, la decisión indelegable del Consejo del SINAES de conceder o no la acreditación oficial, así como las condiciones en que ésta se otorga. En relación con los costos del proceso de acreditación, la fase de evaluación externa tiene un costo de 7.300 dólares, se paga una sola vez cada 4 años. La revisión del informe de avance de cumplimiento tiene un costo de 500 dólares, por lo general se hace una vez al año. Esas tarifas han permanecido invariables desde que SINAES comenzó funciones en 1999. El Estado costarricense asume 2/3 partes del costo total del proceso de acreditación, el tercio restante se cubre con el aporte de las instituciones de educación superior.

\subsubsection{Modelo de Evaluación}

La designación de carrera oficialmente acreditada constituye un reconocimiento público que el SINAES, como único ente facultado para ello por leyes de la República (números 8256 y 8798), brinda a las carreras de las universidades e instituciones parauniversitarias costarricenses. Lo anterior mediante procesos conjuntos y normados, los cuales demuestren de manera fehaciente que las carreras brindan un servicio educativo de calidad y que tienen un compromiso demostrado con la mejora continua. La autoevaluación y la evaluación externa, que conforman 
parte del proceso de acreditación oficial, se ejecutan teniendo como referente obligatorio los criterios establecidos por el Consejo Nacional de Acreditación. El Modelo de evaluación de carreras parauniversitarias es el marco de referencia del proceso de acreditación oficial y comprende no solo el presente documento sino también las guías para carreras parauniversitarias establecidas por el SINAES (Autoevaluación, Evaluación externa, Elaboración y revisión del compromiso de mejoramiento, Elaboración y revisión del avance de cumplimiento del compromiso de mejoramiento y demás guías del SINAES), todas disponibles en la página web del SINAES; estas contienen elementos conceptuales y metodológicos relacionados con las etapas de acreditación, las cuales se presentan como separadas por razones expositivas.

El modelo de evaluación del SINAES procura visualizar, de manera integradora, los principales elementos del proceso educativo: un entorno que contextualiza, unos recursos o insumos necesarios para realizar el proceso educativo, el proceso mismo y unos resultados. Estos elementos, en el modelo del SINAES, se denominan dimensiones y se evalúan tomando como marco de referencia las características propias de la naturaleza de cada carrera por acreditar.

Adicionalmente a las dimensiones, el modelo del SINAES establece cuatro elementos que complementan la evaluación, éstos son los criterios de admisibilidad, los criterios de sostenibilidad de la acreditación y mejoramiento de la carrera y la metaevaluación y la reacreditación.

\subsubsection{Instrumentos de Evaluación}

El Sistema Nacional de Acreditación de la Educación Superior (SINAES) es el órgano oficial de acreditación de la educación superior de Costa Rica, es de carácter nacional y fue creado por Ley de la República No 8256 del 2 de mayo de 2002. Por su naturaleza jurídica, el SINAES está inserto en el sistema de educación superior universitario estatal, es absolutamente independiente y autónomo en sus decisiones académicas y posee la máxima autoridad pública en materia de acreditación de carreras y de programas universitarios. En vinculación con la utilización del nuevo modelo de evaluación para la acreditación oficial de carreras de grado, SINAES ha diseñado un conjunto de cuestionarios dirigidos a estudiantes, docentes, personal administrativo, empleadores (as) y graduados (as). Su diseño requirió tanto el desarrollo de versiones preliminares de los cuestionarios, la validación por medio de expertos, la evaluación cognitiva del ítem y la validación empírica de los instrumentos a través de dos estudios piloto. Estos instrumentos permiten que la carrera recopile el $23 \%$ de la información que apoyará el análisis de las evidencias solicitadas en el modelo de evaluación oficial de carreras. El propósito de la creación de estas herramientas es facilitar la recopilación de información solicitada en el modelo de evaluación, particularmente las 79 evidencias que pueden ser recogidas a través de la aplicación de encuestas.

\subsubsection{Evaluadores}

El SINAES integra un equipo de tres evaluadores externos, conocidos como pares externos, en el que participan expertos nacionales e internacionales (pueden ser dos pares nacionales y uno internacional o dos pares nacionales y uno internacional). Estos profesionales poseen una formación afín a la carrera que será objeto de evaluación. Durante una semana, visitan la carrera a evaluar y realizan una profunda indagatoria in situ, para la cual los pares se reúnen con diversos grupos de la carrera: estudiantes, profesores y autoridades, entre otras reuniones. Al final de la visita, los pares presentan al SINAES un informe en torno a los principales hallazgos e indican su recomendación de acreditación (informe de salida). Para este informe, el SINAES suple el respectivo formato. Los pares evaluadores que provengan del sector empleador no representan a la empresa en la cual laboran, sino que aportan, desde su experiencia, la visión del sector empleador.

\subsubsection{Fases del Proceso}


El proceso es muy exhaustivo y se evalúan más de 170 criterios. A modo de resumen te contamos que hay tres pasos fundamentales: 1. Autoevaluación: quienes integran la carrera hacen una revisión del programa de estudio, infraestructura y calidad de la formación, entre otros elementos. 2. Evaluación externa: un experto nacional y dos internacionales evalúan la calidad de la formación y hacen recomendaciones de mejora 3. Mejora continua: la carrera se compromete a realizar una serie de mejoras que pueden abarcar distintas áreas. La máxima autoridad de la universidad firma un compromiso para cumplir con las mejoras definidas.

\subsubsection{Beneficios de la Acreditación}

La acreditación es un indicativo de fe pública de la calidad de una carrera y de su compromiso con la mejora permanente. La acreditación genera beneficios para los estudiantes, los padres de familia y para los empleadores, autogestores y para las propias instituciones de educación superior. El modelo de acreditación del SINAES cuenta con criterios de calidad, en áreas tales como:

- Actualización del plan de estudios.

- Incorporación de tecnologías en el proceso de formación.

- Regulación de deberes y derechos del personal académico.

- Dotación de personal académico competente.

- Políticas para retención de los mejores académicos.

- Ejecución de un plan de desarrollo para el personal académico e incentivos de promoción.

- Contar con un plan de desarrollo para el personal administrativo.

- Dotación adecuada de infraestructura.

- Acceso a un centro de información y recursos adecuado.

\subsubsection{Incentivos de la Acreditación}

Los beneficios que propone el SINAES a las instituciones de educación en Costa Rica son la Capacitación y la Empleabilidad

- Capacitación

SINAES es una organización con amplia experiencia en ofrecer capacitación a instituciones universitarias y parauniversitarias, carreras de ambos sectores y colaboradores externos. Recomienda que antes de iniciar un proceso de autoevaluación con miras a la acreditación oficial, las instituciones soliciten estas capacitaciones. Los participantes pueden ser diversos según las necesidades de las organizaciones, pero es importante que en las mismas participen las comisiones de autoevaluación, las unidades de calidad, funcionarios a cargo de departamentos institucionales, autoridades, estudiantes, entre otros. Los procesos de capacitación del SINAES son totalmente gratuitos y, en el caso de instituciones y carreras pueden ser solicitados mediante correo electrónico o mediante una nota formal. Para los colaboradores externos, se organiza cursos a lo largo del año. Los colaborares externos interesados en recibir capacitación, pueden igualmente remitir un correo electrónico al SINAES para solicitar información sobre el calendario de capacitaciones.

- Empleabilidad

Cada vez con mayor intensidad la legislación y normativa nacional se enriqueces incorporando el tema de la acreditación. Tanto la legislación, como el Servicio Civil y la Caja Costarricense de Seguro Social incorporan referencias importantes a la acreditación en materia de contratación. Adicionalmente, el SINAES mantiene encuentros con empleadores, tanto del sector público como privado para mantener informados a los directores de Recursos Humanos en de las instituciones y empresas en torno a la importancia de reconocer en las elecciones de 
personal a graduados de carreras acreditadas. Como un estímulo a la acreditación y a los egresados de carreras acreditadas, el Estado Costarricense en la Ley de Fortalecimiento del SINAES No. 8798, publicada en el Diario Oficial La Gaceta en su artículo 4, se establece: "El Estado y sus instituciones procurarán contratar personal graduado de carreras oficialmente acreditadas. Se autoriza al Estado y a sus instituciones para que establezca, en los concursos de antecedentes, las condiciones necesarias para diferenciar entre los graduados de carreras oficialmente acreditadas. En los casos en los que poseer grado académico y título profesional sea requisito de contratación".

\subsection{La Reacreditación}

La acreditación se otorga por un periodo determinado, lo usual es que la primera vez sea por cuatro años. Una vez finalizado ese período, la carrera debe realizar nuevamente la Autoevaluación, así como recibir nuevamente la visita de los dos expertos internacionales y uno nacional (Evaluación externa). Para revalidar su acreditación (reacreditación) la carrera debe demostrar el mejoramiento de su calidad, que se otorga por un plazo de entre dos y ocho años. Así las cosas, la acreditación es un proceso cíclico, la carrera estará en un estado permanente de mejora.

\section{Conclusiones}

Durante mucho tiempo la sociedad no dudaba ni realizaba cuestionamiento de la calidad de las universidades, se tenía como un calificativo agregado por el hecho de haberse constituido como universidad. Sin embargo, con el incremento de apertura de universidades en Panamá y el inicio de los procesos de evaluación y acreditación en Centroamérica y Latinoamérica, comienza a tener relevancia en nuestro país.

El Estudio comparativo de los procesos de evaluación de Costa Rica y Panamá constituyo un análisis amplio de los procesos que iniciaron respectivamente en 1999 y 2011.

Por ende, Costa Rica lleva doce años por delante de Panamá en experiencia realizando estos procesos, por lo que pudimos observar de las normativas, informes y reglamentos aparenta ser más ordenado que nuestro país.

Cada país ha llevado adelante sus procesos y las cifras demuestran que los resultados que han tenido son positivos. Los procesos de evaluación y acreditación de la educación superior son instrumentos para promover el mejoramiento de la calidad de las instituciones de educación superior y de sus programas, así como para ofrecer información pertinente a la sociedad, sobre la calidad de estos.

La acreditación implica un proceso de construcción de consensos sobre criterios, indicadores y estándares de calidad comunes en ambos países, lo cual contribuye a la búsqueda de una visión compartida y de horizontes comunes para el mejoramiento y desarrollo de la educación superior de estos hermanos países.

Debemos aprender de ambos países y lograr el análisis reflexivo de la importancia de estos procesos en la educación de nuestros estudiantes, ya que esto repercutirá en un mundo mejor para todos.

\section{Referencias}

Actualización del informe de autoevaluación para optar por la acreditación ante el Consejo Centroamericano de Acreditación Información adicional para optar por la certificación de alineamiento a la Guidelines of Good Practice de la International Network for Quality Assurance Agencies in Higher Education (INQAAHE). 6 de Agosto del 2009. https://www.sinaes.ac.cr/documentos/Informe_de_Autoevaluacion_para_IN QAAHE.pdf 
Acuerdo No 03-2012. Por el cual se autoriza el pago al Consejo Nacional de Evaluación y Acreditación Universitaria de Panamá -CONEAUPA- de los costos de los trámites y servicios del proceso de evaluación y acreditación.

Barrios, A. d., Castillero, Y., \& Sousa, V. (2020). Evaluación a nivel nacional e internacional de la acreditación superior universitaria en Panamá. Conrado.

Camacho, H., Castillo, D., \& de Franco, M. (2018). La indagación: una estrategia innovadora para el aprendizaje de procesos de investigación. Red de Revistas Científicas de América Latina, el Caribe, España y Portuga.

Código de ética.

Decreto 539 de 30 de agosto de 2019 que reglamenta la Ley 52 de 26 de junio de 2015, por la que se crea el Sistema Nacional de Evaluación y Acreditación para el Mejoramiento de la Calidad de la Educación Superior Universitaria de Panamá

Decreto 539 de 30 de agosto de 2019 que reglamenta la Ley 52 de 26 de junio de 2015, por la que se crea el Sistema Nacional de Evaluación y Acreditación para el Mejoramiento de la Calidad de la Educación Superior Universitaria de Panamá

Dictámen Procuraduría General de la República sobre SINAES 2018

Estado de la Educación Costarricense. Consejo Nacional De Rectores External Review Report According To The Guidelines Of Good

Practices Of Inqaahe Sistema Nacional de Acreditación de la

Educación Superior (SINAES) de Costa Rica. September 2019

Guía Para La Autoevaluación De Carreras - con fines de acreditación oficial - Mayo 2011. https://www.sinaes.ac.cr/documentos/Guia_para_la_Autoevaluacion_para_c arreras_de_grado.pdf

INEC. (2011). Instituto Nacional de Estadística y Censo. Obtenido de Censo Poblacional de la República de Panamá.

Informe de autoevaluación para optar por la acreditación ante el Consejo Centroamericano de Acreditación San José, Costa Rica, 28 de enero del 2008. https://www.sinaes.ac.cr/documentos/Informe_de_Autoevaluacion_para_el_CCA.pdf

Ley 52 de 26 de junio de 2015 que crea el Sistema Nacional de Evaluación y Acreditación para el Mejoramiento de la Calidad de la Educación Superior Universitaria de Panamá y deroga la Ley 30 de 2006.

Ley No 8256. Ley del Sistema Nacional de Acreditación de la Educación Superior (SINAES).

Ley No 8798. Fortalecimiento del Sistema Nacional de Acreditación de la Educación Superior (SINAES).

Manual de uso del sello acreditación No. 32 de 20 de diciembre de 2012: Por la cual se aprueba la Modificación de los Fundamentos Del Modelo de Evaluación y Acreditación Institucional Universitaria de Panamá contenidos en la Resolución 1 Del 1 de diciembre de 2010, Reformado por la Resolución 1 de 25 de marzo de 2011. con el fin de implementar un modelo de evaluación institucional que permita el desarrollo de los procesos con mayor efectividad.

Reglamento de Reconsideración de los Acuerdos Tomados por el Consejo del SINAES en los Procesos de Acreditación.

Reglamento del Consejo Nacional de Acreditación.

Reglamento para el Uso de sellos, Emblemas y Denominación SINAES. Reglamento SINAES

Resolución No. 05 de 5 de marzo de 2020: Por la cual se aprueban los fundamentos del Modelo de Evaluación con fines de Acreditación Institucional.

Resolución No. 06 de 5 de marzo de 2020: Por la cual se aprueba la Matriz de Evaluación y Acreditación Institucional Universitaria de Panamá.

Rosales, I. (2019). La Evaluación en el Nivel Superior. Revista Atlante.

SINAES. (23 de junio de 2019). SINAES. Obtenido de SINAES: https://www.sinaes.ac.cr/index.php/el-proceso 
Terlizzi, M. A. (12 de diciembre de 2014). América Economía. Obtenido de Qué aporta el enfoque sistémico y estratégico a las organizaciones: https://mba.americaeconomia.com/articulos/columnas/que-aporta-el- enfoquesistemico-y-estrategico-las-organizaciones

www.conare.ac.cr Programa Estado De La Nación Apdo. 1174-1200 Pavas, Costa Rica www.estadonacion.or.cr

Tünnermann Bernheim, Carlos. (2008). La calidad de la educación superior y su acreditación: la experiencia centroamericana. Avaliação: Revista da Avaliação da Educação Superior (Campinas), 13(2), 313-336. https://doi.org/10.1590/S1414-40772008000200005

\section{Apéndices}

\section{Tabla $\mathbf{N}^{0} 1$}

Aspectos contemplados en los Modelos de Evaluación 2012 y 2019 de CONEAUPA

\begin{tabular}{|l|l|}
\hline \multicolumn{1}{|c|}{$\mathbf{2 0 1 2}$} & \multicolumn{2}{c|}{$\mathbf{2 0 1 9}$} \\
\hline Obligatorio & Científico y humanístico \\
\hline Flexible & Contextualizado \\
\hline Simple & Flexible \\
\hline Participativo & Participativo \\
\hline Respetuoso de la autonomía universitaria & Permanente \\
\hline Promotor del mejoramiento continuo & Promotor de mejoramiento continuo \\
\hline Transformador & Respetuoso de la autonomía universitaria \\
\hline Científico y humanístico & Respetuoso del acompañamiento continuo \\
\hline Con identidad nacional & \\
\hline Permanente & \\
\hline
\end{tabular}

Fuente: Elaboración propia, a partir de (CONEAUPA,2012) (CONEAUPA, 2020) 